\title{
No Evidence for an Association between JC Polyomavirus Infection and Gastroduodenal Diseases
}

\author{
Azadeh Yazdani Cherati ${ }^{a}$ Yousef Yahyapour ${ }^{b} \quad$ Mohammad Ranaee $^{c}$

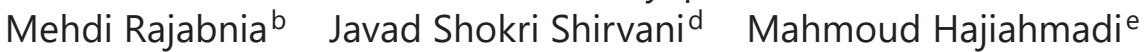 \\ Farzin Sadeghi ${ }^{f}$
}

a Students Research Committee, Babol University of Medical Sciences, Babol, Iran; b Infectious Diseases and Tropical Medicine Research Center, Health Research Institute, Babol University of Medical Sciences, Babol, Iran; ' ${ }^{\mathrm{C}}$ Department of Pathology, Faculty of Medicine, Babol University of Medical Sciences, Babol, Iran; ${ }^{d}$ Department of Internal Medicine, Faculty of Medicine, Babol University of Medical Sciences, Babol, Iran; ${ }^{e}$ Department of Community Medicine, Babol University of Medical Sciences, Babol, Iran; ${ }^{f}$ Cellular and Molecular Biology Research Center, Health Research Institute, Babol University of Medical Sciences, Babol, Iran

\section{Keywords \\ JC polyomavirus - Gastric cancer - Gastroduodenal diseases}

\begin{abstract}
Background: Helicobacter pylori (HP) infection is one of the hypothesized infectious etiologies of gastric cancer (GC) and other gastroduodenal diseases. It was suggested that other infectious agents, including oncogenic viruses, may increase the risk of gastroduodenal diseases. A number of reports regarding JC polyomavirus (JCPyV) have shown that JCPyV could be implicated in colorectal cancer and gastrointestinal carcinogenesis. Objective: The current investigation aimed to investigate whether JCPyV could have any association with the pathogenesis of gastroduodenal diseases either alone or together with HP. Methods: A total of 237 fresh or formalin-fixed and paraffin-embedded gastroduodenal samples were examined by quantitative real-time polymerase chain reaction targeting the JCPyV large tumor antigen (LTag) oncogene, and viral load was determined as viral copy number/cell. Results: In total, 2 out of 237 samples $(0.8 \%)$ were positive for JCPyV LTag DNA. One positive sample derived from diffuse-type gastric adenocarcinoma $\left(6.8 \times 10^{-3}\right.$ copies/cell) and other JCPyV-positive sample obtained from a patient with gastritis $\left(2.5 \times 10^{-3}\right.$ copies/cell) were recorded. Both JCPyV-positive samples were negative for HP infection. Conclusion: This study suggests no association between JCPyV infection and GC or other gastroduodenal diseases. The very low frequency of JCPyV LTag sequences in GC is an important aspect that weakens the hypothesis of the pathogenic role of JCPyV in gastric tumor induction.


Yazdani Cherati et al.: Lack of Association between JCPyV and Gastroduodenal Diseases

\section{Introduction}

Gastroduodenal diseases are a diverse group of medical conditions affecting the stomach and the duodenum. Gastritis (GA), gastric ulcer (GU), duodenal ulcer (DU), and gastric cancer (GC) comprise a significant source of morbidity and mortality worldwide [1]. GC is still the second most frequent cause of cancer death in the world [2, 3]. Gastroduodenal ulcers are regarded as a risk factor for GC, and approximately $2 \%$ of patients with GC showed evidence of GU $[4,5]$. It has been suggested that both genetic and environmental factors play a role in the causation of GC and gastroduodenal diseases. Helicobacter pylori (HP) infection, smoking, dietary factors, and long-term use of nonsteroidal anti-inflammatory drugs are the most important environmental factors associated with an increased risk of gastroduodenal diseases [6].

With respect to infectious etiologies of gastroduodenal diseases, HP infection has been among the most widely investigated. Strong associations between HP infection and gastroduodenal diseases were demonstrated in numerous studies [7]. However, a fraction of gastroduodenal diseases can also occur in the absence of HP infection $[8,9]$. This fact indicates that there must be additional causes of gastroduodenal diseases. Recently, the hypothesis that oncogenic viruses may be correlated with the etiology of GC and gastroduodenal diseases has also been proposed. Throughout the world, approximately $6-16 \%$ of gastric carcinoma cases are associated with Epstein-Barr virus [10]. In addition, several recent reports indicated possible associations between Epstein-Barr virus and gastroduodenal ulcers [11-13].

Recently, a number of reports regarding JC polyomavirus (JCPyV) showed that JCPyV could be implicated in colorectal cancer and gastrointestinal carcinogenesis [10,14]. Infection with JCPyV is ubiquitous, and approximately $90 \%$ of the world's adult population carries antibodies against the virus [15]. There is scarce information about prevalence of JCPyV in the immunocompetent Iranian population. In two separate studies researchers observed that 33 and $38.3 \%$ of healthy individuals excrete JCPyV in their urine $[16,17]$. In addition, there is no information about JCPyV seroprevalence in the immunocompetent Iranian population, and the only reliable seroprevalence study refers to multiple sclerosis patients, in whom the JCPyV seroprevalence was 58.6\% [18]. The mode of JCPyV transmission among individuals remains to be determined. However, the fact that JCPyV has been detected in tonsillar tissue supports a respiratory route [19]. In addition, JCPyV transmission through the gastrointestinal tract by means of feces/urine-contaminated water has also been proposed [20]. The virus has the ability to transform mammalian cells in culture, and the oncogenic potential of JCPyV was established in several animal models [21]. Large tumor antigen (LTag) of JCPyV has been demonstrated to mediate the oncogenic potential of the virus. The expression of JCPyV LTag has been significantly associated with chromosomal instability in a colon cancer cell line [22]. In addition, expression of JCPyV LTag has been demonstrated in a subset of GC samples, and LTag-positive GCs showed a considerable increase in allelic losses and aberrant methylation $[23,24]$. Moreover, according to a recent study, the JCPyV DNA load was significantly higher in GCs than in normal gastric tissue [15]. However, the association of JCPyV with other gastroduodenal lesions that carry a risk of malignant transformation, including GA, GU, and DU, has not been clarified to date.

Thus, the facts reviewed above encouraged us to investigate whether JCPyV could have any association with the pathogenesis of gastroduodenal diseases either alone or together with HP. In this study, we tested 237 fresh or formalin-fixed and paraffin-embedded (FFPE) samples of Iranian patients diagnosed with gastroduodenal diseases (GA, GU, DU, or GC) as well as gastric congested mucosa (CM) from healthy subjects for the presence of JCPyV LTag sequences by quantitative real-time polymerase chain reaction (PCR). 


\section{Materials and Methods}

Clinical Samples

In this cross-sectional study, a total of 237 samples (129 gastroduodenal fresh biopsy samples and 108 FFPE resection specimens) were collected. Out of the 129 gastroduodenal fresh biopsy samples, 52 had a histopathologic GA diagnosis, 20 and 31 samples had a GU and a DU diagnosis, respectively, and 26 samples were diagnosed as GC. Of the 108 FFPE samples, 55 had a GC histopathologic diagnosis and 53 cases were CM from healthy subjects. Gastroduodenal fresh biopsy samples were obtained from patients who underwent endoscopy at the Gastroenterology Department of Ayatollah Rouhani and Shahid Beheshti Hospitals, affiliated to Babol University of Medical Sciences. FFPE resection specimens were collected from the archives of the Pathology Department of Ayatollah Rouhani Hospital affiliated to Babol University of Medical Sciences. Gastroduodenal diseases were diagnosed according to clinical, endoscopic, and histopathologic examinations [13].

\section{DNA Extraction}

Deparaffinization of FFPE resection specimens was done according to a previously described procedure $[25,26]$. Briefly, a number of $5-\mu \mathrm{m}$ tissue sections were collected in a sterile microcentrifuge tube. To dissolve the paraffin, FFPE sections were incubated three times in $500 \mu \mathrm{L}$ of xylene for $10 \mathrm{~min}$ at $60^{\circ} \mathrm{C}$ and were subsequently washed with absolute ethanol. DNA was isolated from fresh and FFPE samples by using the DNA Extraction Mini Kit from Tissue (Yekta TajhizAzma, Tehran, Iran). In brief, for tissue digestion $200 \mu \mathrm{L}$ of tissue lysis buffer and $20 \mu \mathrm{L}$ of proteinase $\mathrm{K}(10 \mathrm{mg} / \mathrm{mL})$ were added to each tube. Samples were subsequently incubated at $60^{\circ} \mathrm{C}$ until the tissue was completely lysed. DNA cleanup was done by mini spin column (silica matrix) according to the manufacturer's instructions. Sterile microcentrifuge tubes containing only reaction mixtures were processed simultaneously with the tissue samples as an extraction-negative control. The quality and quantity of purified DNA was determined using a NanoDrop spectrophotometer (Thermo Scientific, Wilmington, NC, USA). The mean weight of the starting FFPE and fresh tissue samples was $25 \mathrm{mg}$. The yield of DNA for fresh tissue samples was in the range of $~ 100-400 \mathrm{ng} / \mu \mathrm{L}$, while in FFPE tissue samples it was in the range of $\sim 30-70 \mathrm{ng} / \mu \mathrm{L}$. The A260/A280 ratio of eluted DNA from fresh and FFPE tissue samples was $\sim 1.7$, which showed good quality of genomic DNA.

To determine DNA extraction efficiency, a known amount of plasmids carrying the target JCPyV LTag sequence $\left(10^{4}-10^{6}\right.$ copies $/ \mu \mathrm{L}$ of recombinant plasmids) were added to three negative tissue specimens, and total DNA was extracted according to the commercial kit instructions. Recovery of the spiked recombinant plasmids was assessed via quantitative real-time PCR. The ratio of the amount of DNA recovered (quantitated) to the original amount of DNA (known) represents the true extraction efficiency. In our experiment, the average of true DNA extraction efficiency was $30 \%$.

\section{JCPyV Quantitative Real-Time PCR}

The real-time PCR method was utilized to detect and measure the amount of JCPyV LTag DNA load. The JCPyV LTag DNA load was determined as the viral DNA copies/RNase P gene copy (a proven single copy gene), which described the copy number/cell. Construction of plasmids containing cloned target sequences of JCPyV LTag and human RNase P gene (quantitative standards for real-time PCR) was described previously [27, 28]. Quantitative real-time PCR was conducted using a Rotor-Gene ${ }^{\circledR} \mathrm{Q}$ (Qiagen GmbH, Hilden, Germany) real-time PCR system by the primer sets and TaqMan probe specific for the JCPyV LTag gene and the human RNase Pgene according to a previously described procedure $[29,30]$. Each reaction consisted of $100 \mathrm{ng}$ of extracted DNA. To assess the sensitivity of the real-time PCR assay, a standard curve was generated using tenfold dilution series of JCPyV LTag plasmid in genomic extracts obtained from JCPyV-negative fresh and FFPE samples. Dilution experiments using purified plasmid containing JCPyV LTag amplicon showed that the real-time PCR assay invariably detected 50 copies of the JCPyV LTag plasmid on a background of $100 \mathrm{ng}$ of genomic DNA.

\section{Results}

\section{Patient Characteristics}

Among the 237 enrolled participants (mean age $53.3 \pm 16.7$ years, range 15-90 years), 81 (34.2\%) had GC, 51 (21.5\%) had GA, 20 (8.4\%) had GU, and 32 (13.5\%) had DU; 53 samples $(22.4 \%)$ were CM of healthy subjects. Out of the 81 patients with GC, $69(85.2 \%)$ had gastric 
Yazdani Cherati et al.: Lack of Association between JCPyV and Gastroduodenal Diseases

Table 1. Characteristics of the study population according to HP infection

\begin{tabular}{lrrrr}
\hline Variable & HP positive & HP negative & Total & $p$ value \\
\hline Number of patients & $76(33.2 \%)$ & $153(66.8 \%)$ & 229 & \\
Age group & & & & \\
$\quad \leq 55$ years & $44(34.6 \%)$ & $83(65.4 \%)$ & 127 & 0.673 \\
$\quad>55$ years & $32(31.7 \%)$ & $69(68.3 \%)$ & 101 & \\
Sex & & & & 0.204 \\
$\quad$ Male & $47(37.0 \%)$ & $80(63.0 \%)$ & 127 & 0.570 \\
$\quad$ Female & $29(28.4 \%)$ & $73(71.6 \%)$ & 102 & 1.000 \\
Age, years & $54.1(15-86)$ & $52.7(17-90)$ & $53.2(15-90)$ & \\
Smoking status & $10(34.5 \%)$ & $19(65.5 \%)$ & 29 & 0.643 \\
$\quad$ Smokers & $63(33.5 \%)$ & $125(66.5 \%)$ & 188 & \\
$\quad$ Nonsmokers & & & & \\
Alcohol drinking & $9(39.1 \%)$ & $14(60.9 \%)$ & 23 & \\
$\quad$ Yes & $64(33.3 \%)$ & $128(66.7 \%)$ & 192 & \\
$\quad$ No & $13(38.2 \%)$ & $21(61.8 \%)$ & 34 & \\
Family history of GC & $58(33.0 \%)$ & $118(67.0 \%)$ & 176 & \\
$\quad$ Yes & & & & \\
$\quad$ No & $70(40.0 \%)$ & $45(60.0 \%)$ & 75 & \\
Diseases & $7(35.0 \%)$ & $13(65.0 \%)$ & 20 & \\
$\quad$ GC & $38(90.3 \%)$ & $3(9.7 \%)$ & 31 & \\
GU & $11(22.4 \%)$ & $38(77.6 \%)$ & 49 & \\
$\quad$ DU & $0(0 \%)$ & $53(100 \%)$ & 53 & \\
GA & &
\end{tabular}

Values are presented as $n(\%)$ or mean (range). CM, congested mucosa; DU, duodenal ulcer; GA, gastritis; GC, gastric cancer; GU, gastric ulcer; HP, Helicobacter pylori.

adenocarcinoma, intestinal type and $11(13.6 \%)$ had gastric adenocarcinoma, diffuse type. The type of gastric adenocarcinoma was not determined in 1 sample. All GC samples were primary in site. A majority of the samples with GC and GU histopathologic diagnosis were categorized in the age groups $>55$ years, whereas most samples with GA, DU, and CM diagnosis were categorized in the age group $\leq 55$ years. A family history of GC was seen in 21.9 and $25.0 \%$ of the GC and GU groups, respectively, whereas $13.3 \%$ of DU, $10.0 \%$ of CM, and $9.8 \%$ of GA cases had a history of GC in their families. HP infection was detected in $90.3 \%$ of DU, $39.5 \%$ of GC, $35.0 \%$ of GU, and $22.4 \%$ of GA subjects. However, none of the CM samples from healthy subjects were HP positive. Table 1 shows the demographic characteristics of subjects according to HP infection.

\section{Detection and Quantitation of JCPyV}

Human RNase P gene was present in the extracted DNA from all samples (mean RNase P gene copy number: $8.7 \times 10^{5} \pm 1.6 \times 10^{3}$ copies $/ \mu \mathrm{L}$, range $4 \times 10^{3}$ to $7.5 \times 10^{6} \mathrm{copies} / \mu \mathrm{L}$ ). JCPyV occurrence was evaluated by quantitative real-time PCR targeting JCPyV LTag gene. In total, only 2 out of 237 samples ( $0.8 \%)$ were positive for JCPyV LTag DNA. One positive sample belonged to a patient with diffuse-type gastric adenocarcinoma (1 out of $11,9.0 \%$ ), while the other JCPyV-positive sample was obtained from a patient with GA (1 out of 51, 1.9\%). In detail, the first one was a 61-year-old female patient with a gastric adenocarcinoma diagnosis and without a family history of GC. The patient had no history of alcohol drinking and smoking. The JCPyV LTag copy number in this case was $6.8 \times 10^{-3}$ copies/cell. The second patient was 
a 28-year-old man with a GA diagnosis and without a family history of GC. The patient had a history of alcohol consumption and smoking. The GA case showed $2.5 \times 10^{-3}$ copies/cell of JCPyV LTag DNA. Both JCPyV-positive patients were negative for HP infection.

\section{Discussion}

Growing evidence indicates that several common viruses may play an important role as causative agents of human malignancies [31,32]. JCPyV is a ubiquitous virus with oncogenic potential and has been strongly suspected in carcinogenesis throughout the gastrointestinal tract [23, 33-37]. Taking cues from the possible implication of JCPyV LTag in gastrointestinal tumors, in the present study we determined the presence of JCPyV LTag sequences in Iranian patients with GC and other gastroduodenal diseases. Moreover, we evaluated the JCPyV LTagpositive samples in terms of viral copy numbers/cell. In this study only two samples (1 GC and $1 \mathrm{GA}$ ) were positive for JCPyV LTag sequences. Our result reveals a very low frequency of JCPyV LTag sequences in GC and other gastroduodenal diseases, which is inconsistent with a number of other reports $[15,23,36]$. One possible explanation for this inconsistency is the high percentage of FFPE samples in our GC group (67.9\%); the detection rate of JCPyV DNA sequences could be significantly reduced in FFPE samples $[15,38]$. Another plausible explanation could be geographic and ethnic variations in JCPyV distribution. The JCPyV LTag sequence was detected in $26.0 \%$ of Tunisian GC patients [36]. In addition, $57.0 \%$ of Korean [23] and $86.3 \%$ of Japanese [15] GC patients harbored the JCPyV LTag sequence in their neoplastic tissues. In addition, to address the sensitivity of our assay, the forward JCPyV primer contains a mismatch (T/C) in position 7 that matches different JCPyV variants isolated in Asia, including isolates identified in Iran (GenBank AKX816852 and KJ719314). In the present investigation, low copy numbers of JCPyV LTag gene/cell were detected in our two positive samples. In our opinion, low copy numbers of JCPyV genome in gastric tissue weaken the hypothesis of a pathogenic role of JCPyV in tumor induction and might be explained by simple persistent viral replication as a passenger virus during the development of GC. In addition, low copy number of JCPyV may indicate unsuccessful viral replication largely due to nonfunctional LTag.

Despite this, further limitations regarding the present investigation should be highlighted, including the retrospective setting and the high percentage of FFPE samples in our GC group. Moreover, there is an absence of $\mathrm{p} 53 / \mathrm{pRb}$ SNP data in the Iranian population and also an absence of LTag sequence variations in the endemic JCPyV.

Taken together, the present study provides the first data on the prevalence of JCPyV in Iranian patients with GC and other gastroduodenal diseases. The current investigation suggests no association between JCPyV infection and gastroduodenal diseases. More worldwide epidemiological studies should be done to ascertain the role of JCPyV in gastric carcinogenesis.

\section{Acknowledgments}

The authors would like to acknowledge the support of the directors and staff of the Gastroenterology Department of Ayatollah Rouhani and Shahid Beheshti Hospitals and the Pathology Department of Ayatollah Rouhani Hospital affiliated to Babol University of Medical Sciences for their collaboration in sample collection. This study was financially supported by a grant from Babol University of Medical Sciences (project code: 9503117). 
Yazdani Cherati et al.: Lack of Association between JCPyV and Gastroduodenal Diseases

\section{Statement of Ethics}

This study was approved by the Ethics Committee of Babol University of Medical Sciences. Written informed consent was obtained from all subjects.

\section{Disclosure Statement}

The authors declare that they have no conflict of interest.

\section{References}

1 Gillen D, McColl KE: Gastroduodenal disease, Helicobacter pylori, and genetic polymorphisms. Clin Gastroenterol Hepatol 2005;3:1180-1186.

2 Parkin DM, et al: Global cancer statistics, 2002. CA Cancer J Clin 2005;55:74-108.

3 Jemal A, et al: Cancer statistics, 2004. CA Cancer J Clin 2004;54:8-29.

4 Lee TY, et al: A tool to predict risk for gastric cancer in patients with peptic ulcer disease on the basis of a nationwide cohort. Clin Gastroenterol Hepatol 2015;13:287-293.e1.

5 Hansson LE: Risk of stomach cancer in patients with peptic ulcer disease. World J Surg 2000;24:315-320.

6 Karimi P, et al: Gastric cancer: descriptive epidemiology, risk factors, screening, and prevention. Cancer Epidemiol Biomarkers Prev 2014;23:700-713.

7 Helicobacter and Cancer Collaborative Group: Gastric cancer and Helicobacter pylori: a combined analysis of 12 case control studies nested within prospective cohorts. Gut 2001;49:347-353.

8 Iijima K, et al: Helicobacter pylori-negative, non-steroidal anti-inflammatory drug: negative idiopathic ulcers in Asia. World J Gastroenterol 2014;20:706-713.

9 Kato S, et al: Helicobacter pylori infection-negative gastric cancer in Japanese hospital patients: incidence and pathological characteristics. Cancer Sci 2007;98:790-794.

10 Selgrad M, et al: The role of viral and bacterial pathogens in gastrointestinal cancer. J Cell Physiol 2008;216: 378-388.

11 Saxena A, et al: Association of Helicobacter pylori and Epstein-Barr virus with gastric cancer and peptic ulcer disease. Scand J Gastroenterol 2008;43:669-674.

12 Shukla SK, et al: Epstein-Barr virus DNA load and its association with Helicobacter pylori infection in gastroduodenal diseases. Braz J Infect Dis 2011;15:583-590.

13 Cardenas-Mondragon MG, et al: Epstein-Barr virus association with peptic ulcer disease. Anal Cell Pathol (Amst) 2015;2015:164840.

14 Delbue S, Comar M, Ferrante P: Review on the role of the human Polyomavirus JC in the development of tumors. Infect Agent Cancer 2017;12:10.

15 Murai Y, et al: High JC virus load in gastric cancer and adjacent non-cancerous mucosa. Cancer Sci 2007; 98: 25-31.

16 Bozorgi SM, et al: Molecular prevalence of JC virus in Tehran, Iran. Gastroenterol Hepatol Bed Bench 2012;5: 84-89.

17 Behzad-Behbahani A, et al: Detection of BK virus and JC virus DNA in urine samples from immunocompromised (HIV-infected) and immunocompetent (HIV-non-infected) patients using polymerase chain reaction and microplate hybridisation. J Clin Virol 2004;29:224-229.

18 Alroughani R, et al: JC virus seroprevalence and seroconversion in multiple sclerosis cohort: a Middle-Eastern study. J Neurol Sci 2016;360:61-65.

19 Imperiale MJ: Oncogenic transformation by the human polyomaviruses. Oncogene 2001;20:7917-7923.

20 Bofill-Mas S, et al: Potential transmission of human polyomaviruses through the gastrointestinal tract after exposure to virions or viral DNA. J Virol 2001;75:10290-10299.

21 White MK, Khalili K: Polyomaviruses and human cancer: molecular mechanisms underlying patterns of tumorigenesis. Virology 2004;324:1-16.

22 Ricciardiello L, et al: Induction of chromosomal instability in colonic cells by the human polyomavirus JC virus. Cancer Res 2003;63:7256-7262.

23 Shin SK, et al: Oncogenic T-antigen of JC virus is present frequently in human gastric cancers. Cancer 2006; 107:481-488.

24 Yamaoka S, et al: Genetic and epigenetic characteristics of gastric cancers with JC virus T-antigen. World J Gastroenterol 2009;15:5579-5585.

25 Yahyapour Y, et al: Detection of Merkel cell polyomavirus and human papillomavirus in esophageal squamous cell carcinomas and non-cancerous esophageal samples in northern Iran. Pathol Oncol Res 2016;22:667-672.

26 Yahyapour Y, et al: Evaluation of human papilloma virus infection in patients with esophageal squamous cell carcinoma from the Caspian Sea area, north of Iran. Asian Pac J Cancer Prev 2012;13:1261-1266. 
27 Sadeghi F, et al: Detection of Merkel cell polyomavirus large T-antigen sequences in human central nervous system tumors. J Med Virol 2015;87:1241-1247.

28 Sadeghi F, et al: Prevalence of JC polyomavirus large T antigen sequences among Iranian patients with central nervous system tumors. Arch Virol 2015;160:61-68.

29 MacKenzie J, et al: Association between simian virus 40 DNA and lymphoma in the United Kingdom. J Natl Cancer Inst 2003;95:1001-1003.

30 Imajoh M, et al: Novel human polyomaviruses, Merkel cell polyomavirus and human polyomavirus 9, in Japanese chronic lymphocytic leukemia cases. J Hematol Oncol 2012;5:25.

31 Ravart M, Cote H: Sexoanalysis: a new insight-oriented treatment approach for sexual disorders. J Sex Marital Ther 1992;18:128-140.

32 Damania B: DNA tumor viruses and human cancer. Trends Microbiol 2007;15:38-44.

33 Casini B, et al: Presence and incidence of DNA sequences of human polyomaviruses BKV and JCV in colorectal tumor tissues. Anticancer Res 2005;25:1079-1085.

34 Del Valle L, et al: Detection of JC virus DNA sequences and expression of viral T antigen and agnoprotein in esophageal carcinoma. Cancer 2005;103:516-527.

35 Hori R, et al: Detection of JC virus DNA sequences in colorectal cancers in Japan. Virchows Arch 2005; 447: 723-730.

36 Ksiaa F, et al: The presence of JC virus in gastric carcinomas correlates with patient's age, intestinal histological type and aberrant methylation of tumor suppressor genes. Mod Pathol 2010;23:522-530.

37 Theodoropoulos G, et al: Assessment of JC polyoma virus in colon neoplasms. Dis Colon Rectum 2005;48: 86-91.

38 Ricciardiello L, et al: JC virus DNA sequences are frequently present in the human upper and lower gastrointestinal tract. Gastroenterology 2000;119:1228-1235. 